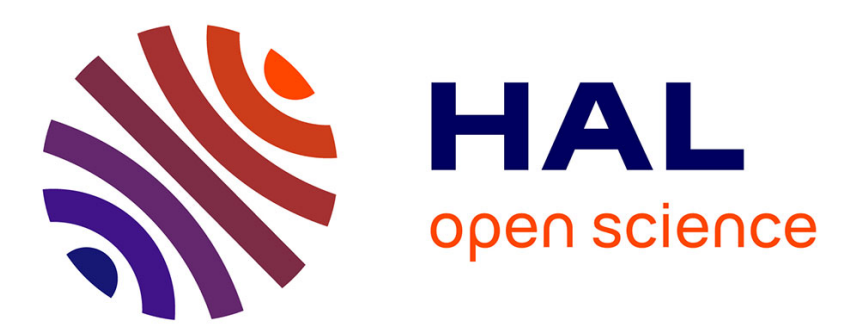

\title{
High Tc SQUIDs for Unshielded Measuring in Disturbed Environments
}

\author{
V. Schultze, R. Ijsselsteijn, R. Stolz, V. Zakosarenko
}

\section{To cite this version:}

V. Schultze, R. Ijsselsteijn, R. Stolz, V. Zakosarenko. High Tc SQUIDs for Unshielded Measuring in Disturbed Environments. Journal de Physique IV Proceedings, 1996, 06 (C3), pp.C3-367-C3-372. 10.1051/jp4:1996356 . jpa-00254274

\section{HAL Id: jpa-00254274 https://hal.science/jpa-00254274}

Submitted on 1 Jan 1996

HAL is a multi-disciplinary open access archive for the deposit and dissemination of scientific research documents, whether they are published or not. The documents may come from teaching and research institutions in France or abroad, or from public or private research centers.
L'archive ouverte pluridisciplinaire HAL, est destinée au dépôt et à la diffusion de documents scientifiques de niveau recherche, publiés ou non, émanant des établissements d'enseignement et de recherche français ou étrangers, des laboratoires publics ou privés. 


\title{
High $T_{\mathrm{c}}$ SQUIDs for Unshielded Measuring in Disturbed Environments
}

\author{
V. Schultze, R. Ijsselsteijn, R. Stolz and V. Zakosarenko \\ Institut fiur Physikalische Hochtechnologie e.V., PF 100239, 07702 Jena, Germany
}

\begin{abstract}
Directly coupled dc SQUID gradiometers on the basis of $\mathrm{YBa}_{2} \mathrm{Cu}_{3} \mathrm{O}_{7-\mathrm{x}}$ for unshielded NDE measurements are investigated. The influence of different SQUID layout parameters on the field sensitivity is shown. A sensitivity of $500 \mathrm{fT} /(\mathrm{cm} \cdot \sqrt{ } \mathrm{Hz})$ is achieved with a baseline of $3.6 \mathrm{~mm}$. A slit in an aluminium plate was detected in an demonstration of NDE in $1 \mathrm{~cm}$ depth with eddy current method.
\end{abstract}

\section{INTRODUCTION}

The ultimate sensitivity of SQUTDs to magnetic flux results not only in applications for exclusive purposes, but they are also established in biomagnetic measurements and emerging in nondestructive evaluation (NDE) [1-3]. Because of the high costs wider applications were restricted as long as liquid helium for cooling and especially shielded rooms to protect the SQUID from environmental disturbances were needed. The comparable cheap coolant liquid nitrogen used for devices made of high temperature superconductors raised new interest to apply SQUIDs in lower cost measurement systems, thus outside shielded rooms.

The purpose of our work is to develop high $T_{c}$ dc-SQUIDs for NDE of deep defects in the millimetre or centimetre range. The SQUIDs have to be able to operate without shielding in conventional electromagnetically disturbed environments.

NDE offers the possibility to use gradiometer structures which remain sensitive to near sources of magnetic fields (defects in the investigated material) and suppress external magnetic disturbances which are nearly homogeneous because of their long distance from the SQUID. Due to the skin effect in the materials to be investigated (steel or aluminium) NDE of deep defects demands a high field gradient sensitivity in the 1 or $10 \mathrm{~Hz}$ frequency range. This requires a low flux noise of the SQUTD structure in this frequency range and a large effective area. Furthermore the baseline of the gradiometer should be comparable to the distance of the SQUID to the defects to achieve best sensitivity [4].

A large effective area can most easily be achieved with a large bare SQUID. However this way is restricted because of the SQUID inductance which should not exceed $100 \mathrm{pH}$ at $77 \mathrm{~K}$ [5]. For this reason it is advantageous to use a separate pickup coil having a large effective flux capture area. To avoid inductance mismatch between the SQUID and pickup coil it can be coupled to the SQUID via a multiturn spiral input coil. The SQUID is usually formed as a washer [6]. These complex structures have already been realised in high $T_{c}[7,8]$, but the fabrication is still difficult. Such devices usually exhibit substantial $1 / f$ noise which is drastically enhanced in unshielded operation. This also holds for devices where SQUID and pickup coil with flux transformer are fabricated on separate chips and afterwards pressed together in flip chip configuration [9]. 
Therefore single layer devices are still attractive for SQUID designs. This can be a large washer that takes advantage of the flux focusing effect [10] without increasing the SQUID inductance. For magnetometers with this concept a good field resolution of $170 \mathrm{fT} / \sqrt{ } \mathrm{Hz}$ down to frequencies of $1 \mathrm{~Hz}$ was achieved [11], but for gradiometers the slit in the washers would increase the inductance unacceptably. Another approach is to couple a large gradiometer pickup coil directly to the SQUID [12-16]. The SQUID as a galvanometer detects the current originated by the field in the pickup loop. Although for this layout the inductance mismatch between pickup coils and SQUID will be substantial, acceptable effective areas can be achieved. Field resolutions of such directly coupled magnetometers (or also called "galvanometers") of $26 \mathrm{fT} / \sqrt{ } \mathrm{Hz}$ at $1 \mathrm{~Hz}$ are reported [17].

We investigated a directly coupled gradiometer SQUTD. Its design will be described in section 2 . In section 3 and 4 we report on the fabrication technology and obtained results for several gradiometers, respectively. Especially, first results in a NDE application will be presented.

\section{DESIGN}

The layout of the single layer parallel gradiometer SQUD is shown in Fig. 1. In the central line of the gradiometer loop there are four SQUIDs in series. To study the influence of the SQUID inductance on the performance of the gradiometer, various SQUID loop sizes were used.
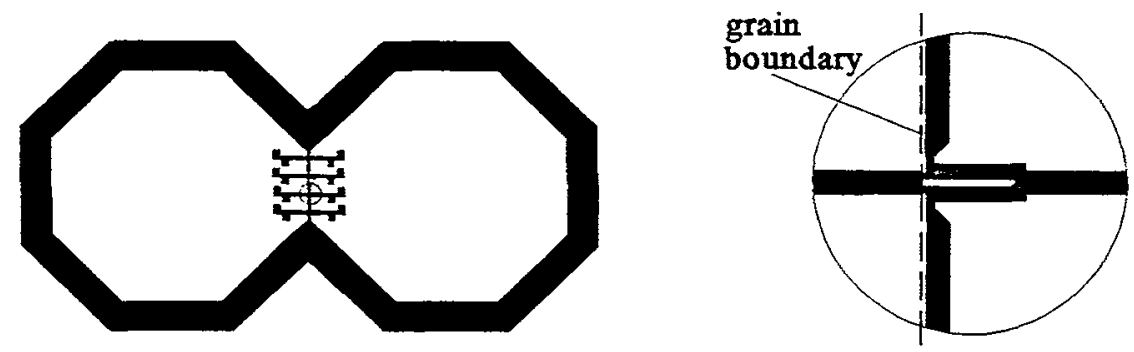

Fig. 1. View of a SQUID gradiometer (left) and a SQUID in the middile of the structure (right).

The layout of this structure was designed in the following way: For a gradiometer the figure of merit is the field gradient sensitivity $\partial B_{m i n} / \partial x$. It is supposed to result from the rms voltage noise $\checkmark S_{V}$ of the SQUID

$$
\frac{\partial B_{\min }}{\partial x}=\frac{2 \sqrt{S_{B}}}{b}=\frac{2 \sqrt{S_{V}}}{b A_{\text {eff }} V_{\Phi}}
$$

where $\widehat{S}_{B}$ is the rms magnetic field noise in each pickup loop with an effective area $A_{\text {eff }} b$ is the gradiometer baseline, and $V_{\Phi}=\partial V / \partial \Phi$ is the flux-to-voltage transfer function. Taking the Josephson junction resistance as the main noise source, thus neglecting critical current fluctuations and flux noise of poor quality films, we have

$$
S_{V}=16 k_{B} T R
$$


with $R$ the junction resistance and $T$ the operation temperature [18]. $V_{\phi}$ degrades with temperature in accordance with [19]

$$
V_{\Phi}=\frac{4}{\Phi_{0}} \cdot \frac{I_{c} R}{1+\beta} \exp \left[-3.5 \pi^{2} \frac{k_{B} T \cdot L_{S Q}}{\Phi_{0}^{2}}\right]
$$

where $\beta=2 L_{S Q} I_{c} / \Phi_{0}$ is the SQUID parameter, $L_{S Q}$ the SQUID inductance, $I_{c}$ the critical current of one Josephson junction, and $\Phi_{0}$ the flux quantum.

So, to get a low magnetic flux noise the junctions should have a high $I_{c} R$ product, and the SQUID should have a low inductance. $L_{S Q}$ and $I_{c}$ on the other hand must be balanced to have $\beta$ not much larger than I [20]. Additionally the influence of the SQUID inductance on the effective area

$$
A_{\text {eff }}=2 A_{p u} \frac{k L_{S Q}}{2 k L_{S Q}+L_{p u}}
$$

must be taken into account, where $A_{p u}$ and $L_{p u}$ are the pickup area and the inductance of one gradiometer loop, respectively. $k<1$ represents the part of the SQUID loop where the current from the pickup loops flows. The inductance of the rectangular SQUID was estimated with three different formulas: for coplanar lines [21], for a round washer, and for a quadratic washer [6], where the rectangular SQUTD hole was approximated by a quadratic or a round one having the same area. Contributions of kinetic inductances were also included assuming a London penetration depth of $300 \mathrm{~nm}$. The results of the three methods agree within $10 \%$. The optimisation of $A_{\text {eff }}$ by given $L_{S Q}$ and chip size is reported in [22].

The SQUID structures are designed for $10 \times 10 \mathrm{~mm}^{2}$ substrates. The pickup loops have an outer diameter of $4 \mathrm{~mm}$. Their line width of $400 \mu \mathrm{m}$ was chosen to be sure that the superconducting loop should be able to carry the screening current originated by moving the SQUID unshielded in the earth magnetic field. The gradiometer baseline $b$ is $3.6 \mathrm{~mm}$. The SQUID inductance is built by a U-form strip of $20 \mu \mathrm{m}$ width having a $10 \mu \mathrm{m}$ slit. The length $l$ of this slit was varied to minimise the magnetic field noise $\sqrt{ } S_{B}$. Figure 2 shows the calculated dependence of $\checkmark S_{B}$ on $l$ for different critical currents $I_{c}$ of the junctions assuming the $I_{c} R$ product to be $100 \mu \mathrm{V}$. The values of the SQUID parameter $\beta$ are shown too.

To be able to use a SQUD which is near the optimum performance for a given $I_{c}$ the four SQUIDs got hole lengths of

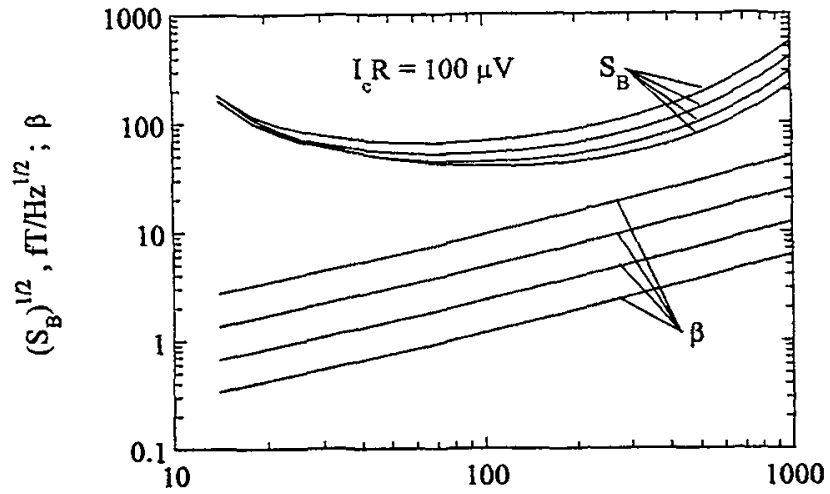

Length of the SQUID loop, $\mu \mathrm{m}$

Fig. 2. Field noise and SQUID parameter $\beta$ calculated for $I_{\mathrm{c}}=20$ (lower-), 40, 80, and $160 \mu \mathrm{A}$ (upper curve). $40,80,120$ and $160 \mu \mathrm{m}$. 


\section{FABRICATION}

The SQUID structures were realised using symmetrical $24^{\circ}$ (100) $\mathrm{SrTiO}_{3}$ bicrystals. A $100 \mathrm{~nm}$ thick $\mathrm{YBa}_{2} \mathrm{Cu}_{3} \mathrm{O}_{7-\mathrm{x}}$ layer was deposited, either by hollow cathode sputter deposition (HC) or by pulsed laser deposition (PLD). The critical current densities at $77 \mathrm{~K}$ for the HC and PLD layers are up to $2 \cdot 10^{6} \mathrm{~A} / \mathrm{cm}^{2}$ and $3 \cdot 10^{6} \mathrm{~A} / \mathrm{cm}^{2}$, respectively. Directly after $\mathrm{YBa}_{2} \mathrm{Cu}_{3} \mathrm{O}_{7-\mathrm{x}}$ deposition a $100 \mathrm{~nm}$ thick gold layer was evaporated. After removing gold and $\mathrm{YBa}_{2} \mathrm{Cu}_{3} \mathrm{O}_{7-\mathrm{x}}$ film at the substrate edges the $\mathrm{SrTiO}_{3}$ was shortly etched with diluted $\mathrm{HF}$ to make the grain boundary visible. Next $\mathrm{YBa}_{2} \mathrm{Cu}_{3} \mathrm{O}_{7-\mathrm{x}}$ and gold were patterned using $\mathrm{Ar}$ ion beam etching through a photoresist mask. The width of the junctions was about $7 \mu \mathrm{m}$.

\section{RESULTS}

All results were achieved at $77 \mathrm{~K}$.

The Josephson junctions of the SQUIDs showed $I_{c} R$ products of $(70 \ldots 150) \mu \mathrm{V}$ for the HC structures and (180...240) $\mu \mathrm{V}$ for the PLD structures. Critical currents of the junctions ranged from (26...153) $\mu \mathrm{A}$ for $\mathrm{HC}$ and (95...330) $\mu \mathrm{A}$ for PLD structures. Because of the large critical currents only the smaller SQUID loops with 40 and $80 \mu \mathrm{m}$ length could be used. For the other SQUTDs $\beta$ was too large and with that the voltage modulation too small to get stable working regimes. In the following the results of two HC and two PLD structures will be presented.

The $V(I)$ - and $V(\Phi)$ - curves as well as noise spectra were measured for all SQUDDs. Noise measurements were performed in flux-locked loop at a bias current yielding maximum $V_{\Phi}$. The flux modulation frequency was $125 \mathrm{kHz}$. Additionally bias reversal with $4 \mathrm{kHz}$ could be used. Shielded measurements were performed with two concentric mu-metal cylinders. Fig. 3 shows examples of noise spectra.

To determine the magnetic field sensitivity $\mathfrak{S}_{B}$ and the effective and parasitic areas the SQUIDs were used without shielding in a plastic cryostat. $A_{e f f}$ was determined by changing the distance of a small coil outside the cryostat to the SQUID. $A_{\text {par }}$ as a value of the unwanted response of the gradiometer to homogeneous fields was measured in the field of a large Helmholtz coil system having a deviation from homogeneity $<2 \cdot 10^{-4}$.

The parameters of the measured SQUIDs are summarised in Table 1 . The values of the critical current are obtained from the $V(I)$ characteristics taking into account the thermal noise rounding.

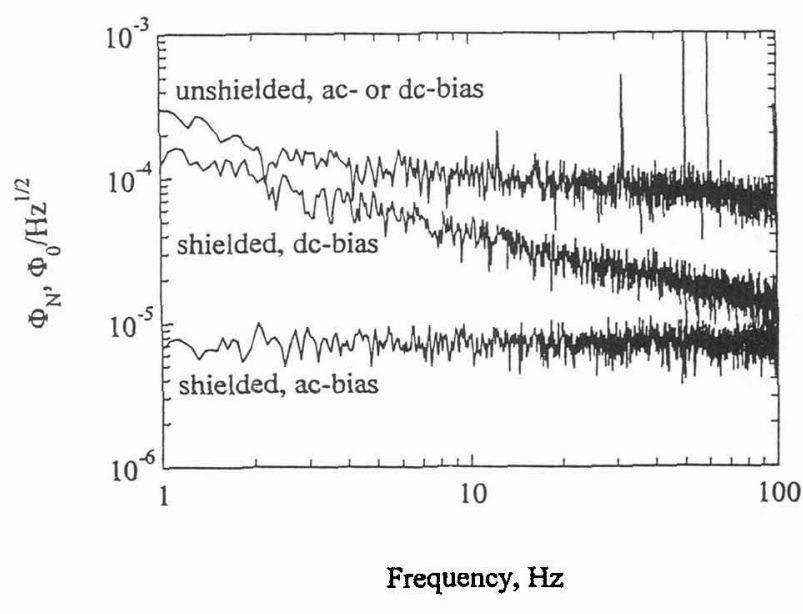

Fig. 3. The noise spectra of SQUID \#1 measured in magnetical shieldings with dc- and ac-bias and without shielding. 
Table 1: Parameters of the SQUIDs (symbols are explained in the text).

\begin{tabular}{|c|c|c|c|c|}
\hline SQUID \# / fabrication & $1 / \mathrm{HC}$ & $2 / \mathrm{HC}$ & $3 /$ PLD & 4 / PLD \\
\hline$l / \mu \mathrm{m}$ & 40 & 80 & 40 & 40 \\
\hline$I R / \mu \mathrm{V}$ & 105 & 20 & 230 & 240 \\
\hline$I_{c}$ of one junction $/ \mu \mathrm{A}$ & 65 & 15 & 88 & 87 \\
\hline$V_{\Phi} / \mu V / \Phi_{0}(\exp . /$ calc. $)$ & $38 / 100$ & $24 / 29$ & $78 / 177$ & $85 / 107$ \\
\hline $\begin{array}{l}\sqrt{ } \mathrm{S}_{\Phi} / \mu \Phi_{0} / \sqrt{ } \mathrm{Hz} \\
(\text { exp., dc-bias, @200 Hz) }\end{array}$ & 10 & 8 & 15 & 15 \\
\hline 1/f- onset / Hz (dc-bias ) & $>1 \mathrm{k}$ & 100 & $>\mathbf{l k}$ & $>\mathrm{lk}$ \\
\hline $\begin{array}{l}\sqrt{S_{\Phi}} \mu \Phi_{0} / \sqrt{ } \mathrm{Hz} \\
\text { (exp., ac-bias, white) }\end{array}$ & 7 & 30 & -- & 6 \\
\hline$S_{\Phi} / \mu \Phi_{\sigma} / \sqrt{ } \mathrm{Hz}$ (calc) & 2 & 5 & 2 & 1 \\
\hline$A_{\text {eff }} / \mathrm{mm}^{2}$ (exp. $/$ calc. $)$ & $0.060 / 0.064$ & $0.175 / 0.116$ & $0.057 / 0.064$ & $--/ 0.064$ \\
\hline$\$_{\mathrm{B}} / \mathrm{fT} / \sqrt{\mathrm{Hz}} @ 200 \mathrm{~Hz}$ & 227 & 95 & 487 & 207 \\
\hline$\partial B_{\min } / \partial x / \mathrm{pT} /(\mathrm{cm} \sqrt{H z}) @ 200 \mathrm{~Hz}$ & 1.3 & 0.5 & 2.7 & 1.1 \\
\hline$A_{\mathrm{par}} / \mu \mathrm{m}^{2}$ & 589 & - & 517 & - \\
\hline
\end{tabular}

The results show that except for SQUID \#3 reasonable good magnetic flux noise $\sqrt{S_{\phi}}$ could be achieved. Without shielding the noise level increases approximately by one order of magnitude. Best field resolution showed SQUID \#2. Here the low critical current allowed to use a larger SQUID loop that gives a larger effective area. To take further advantage of the larger SQUID loops the junction width should be reduced to about $1 \mu \mathrm{m}$ to have small critical currents and still a high $I R$ product. The large parasitic areas in the sensitive direction of the gradiometer are originated by the magnetometer structure of the SQUID itself.

With SQUID \#3 first eddy current NDE measurements were performed. Fig. 4 shows the experimental setup. We tried to detect a $4 \mathrm{~cm}$ long, $1 \mathrm{~mm}$ wide slit made in a $4 \mathrm{~mm}$ thick $400 \times 400 \mathrm{~mm}^{2}$ plate of $99.5 \%$ aluminium. The plate with the slit was placed at different distances to the SQUID and was covered with other Al plates. A straight wire carrying an ac current was used for excitation of eddy currents. In Fig. 5 the results of the measurements are given which show the principal possibility to detect defects in various depths with our high $T_{c}$ SQUID gradiometer.

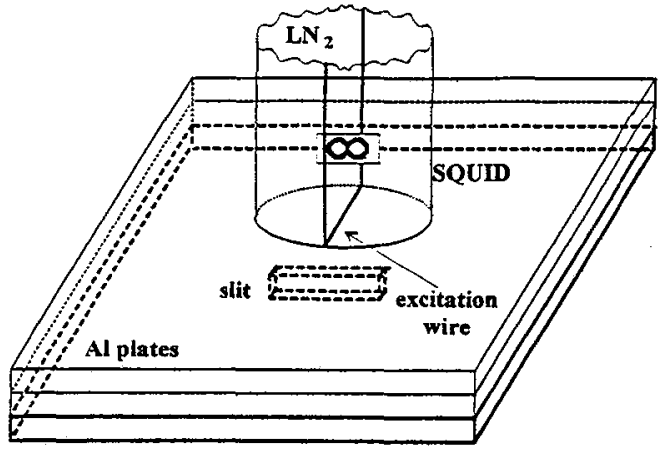

Fig. 4. Experimental setup for eddy current NDE of a phantom object, i. e., an aluminium plate with a slit. 


\section{Summary}

An integrated single layer YBCO SQUID gradiometer was developed and manufactured for unshielded non-destructive evaluation of deep defects in metal. A sensitivity of 500 $\mathrm{fT} / \mathrm{cmHz}^{1 / 2}$ was achieved. A demonstration of NDE with the eddy current method shows the possibility to detect a slit laying under the surface of an $\mathrm{Al}$ plate.

This work was partially supported by the German BMBF in the HOTRONIK program.

\section{References}

[1] Wikswo J.P. Jr., IEEE Trans. Appl. Supercond. 5 (1995) 74-120

[2] Weinstock H., Physica C 209 (1993) 269-272

[3] Carelli P. and Pizzella V., Supercond Sci Technol. 5 (1992) 407-420

[4] Donaldson G.B., Cochran A and Bowman R.M., The New Superconducting Electronics (Kluwer Academic Publishers, Dordrecht, Boston, London, 1993) pp.181-220

[5] Friedl G. et al., IEEE Trans. Appl. Supercond. 3 (1993) 2018 2021

[6] Jaycox J.M., Ketchen M.B., IEEE Trans. Magnetics MAG-17 (1981) 400-403

[7] David B. et al., "High- $T_{c}$ SQUDD Magnetometers for Biomagnetic Measurements", 5th ISEC, Nagoya 18-21 September 1995, Extended Abstracts pp. 329-331

[8] Fife A.A. et al., IEEE Trans. Appl. Supercond 5 (1995) 3113-3118

[9] Dantsker E. et al., Appl. Phys. Lett. 67 (1995) 725-727

[10] Ketchen M.B. et al., "DC SQUID flux focuser", SQUID '85, Berlin 25-28 June, H.D.Hahlbohm and H.Lübbig Ed. (Walter de Gruyter \&Co., Berlin, New York) pp. 865871

[11] Zhang Y. et al., IEEE Trans. Appl. Supercond. 3 (1993) 2465-2468

[12] Daalmans G.M. et al., IEEE Trans. Appl. Supercond. 5 (1995) $3109-3112$

[13] Koelle D. et al., Appl. Phys. Lett. 63 (1993) 2271-2273

[14] Zakosarenko V. et al., Appl. Phys. Lett. 65 (1994) 779-781

[15] Lee L.P. et al., Appl. Phys. Lett. 66 (1995) 1539-1541

[16] Park K. et al., IEEE Trans. Appl. Supercond. 5 (1995) 3119-3122

[17] Cantor R et al., IEEE Trans. Appl. Supercond. 5 (1995) 2927-2930

[18] Bruines J.J.P., de Waal V.J. and Mooij J.E., J. Low Temp. Phys. 46 (1982) 383-393

[19] Enpuku K., Shimomura Y. and Kisu T., J. Appl. Phys. 73 (1993) 7929-7934

[20] Tesche C.D. and Clarke J., J. Low Temp. Phys. 29 (1977) 301-321

[21] Zinke O. and Seither H., "Widerstände, Kondensatoren, Spulen und ihre Werkstoffe" (Springer, Berlin, 1982)

[22] Zakosarenko V. et al., "Integrated LTS Gradiometer SQUDD Systems for Unshielded Measurements in Disturbed Environment", 5th ISEC, Nagoya 18-21 September 1995, Extended Abstracts pp. 355-357 